\title{
Transport Study of LHD High-Beta Plasmas Based on Power Balance Analysis with TASK3D Code Module*)
}

\author{
Ryosuke SEKI, Hisamichi FUNABA, Kiyomasa WATANABE, Satoshi OHDACHI, \\ Yasuhiro SUZUKI, Satoru SAKAKIBARA, Masahiko SATO, Masayuki YOKOYAMA, \\ Sadayoshi MURAKAMI ${ }^{1)}$, Atsushi FUKUYAMA ${ }^{1)}$ and Noriyoshi NAKAJIMA \\ National Institute for Fusion Science, Toki 509-5292, Japan \\ ${ }^{1)}$ Graduate School of Engineering Kyoto University, Kyoto 606-8501, Japan
}

(Received 22 December 2010 / Accepted 22 April 2011)

\begin{abstract}
We customized TASK3D in order to add a new function, TR-snap, for evaluating transport coefficients on the basis of a power balance analysis. To check the validity of the TR-snap's results, the thermal transport coefficient evaluated by TR-snap was compared with results of PROCTR, which is used as the transport analysis code in the Large Helical Device (LHD). The thermal transport coefficient evaluated by TR-snap was almost the same as that of PROCTR. The effect of re-entering fast ions on the transport coefficients in a typical LHD high-beta plasma was investigated using customized TASK3D. When the effect of the re-entering fast ions on the neutral-beam injection heating efficiency is considered, the thermal transport coefficient is slightly larger than that without considering the re-entering fast ions. However, the tendency of the thermal transport coefficient with considering the re-entering fast ions is rarely different from the previously identified tendency.
\end{abstract}

(C) 2011 The Japan Society of Plasma Science and Nuclear Fusion Research

Keywords: transport analysis code, power balance analysis, TASK3D, re-entering fast ion, high-beta plasma

DOI: $10.1585 /$ pfr.6.2402081

\section{Introduction}

In the Large Helical Device (LHD), the volumeaveraged beta $\langle\beta\rangle$ reached $5 \%$ at a low magnetic field when the tangential neutral beam injectors (NBIs) were used [1]. In many experiments, the plasma confinement properties of the high-beta plasmas have been investigated using transport analyses. The performance of the global energy confinement time has been shown to degrade with the volume-averaged beta in the LHD high-beta plasma [2]. To investigate the plasma confinement properties in more detail, it is necessary to evaluate the transport coefficients in more experiments. Thus, we need a convenient transport coefficient evaluation tool.

On the other hand, an integrated transport code for three dimensional configurations (TASK3D) has been developed in order to systematically clarify the confinement physics in a toroidal helical plasma. TASK3D is mainly based on a transport simulation combining various simplified models describing physical processes in different hierarchies. It is being developed on the basis of an integrated transport code for tokamak plasmas, TASK [3] (Transport Analyzing System for tokamaK), which was developed in Kyoto University. TASK3D has a modular structure, as shown in Fig. 1, which allows us to conduct simulations using an individual module or a combination of modules as required. TASK3D enables the prediction of the time

author'se-mail: seki.ryohsuke@lhd.nifs.ac.jp

*) This article is based on the presentation at the 20th International Toki Conference (ITC20).

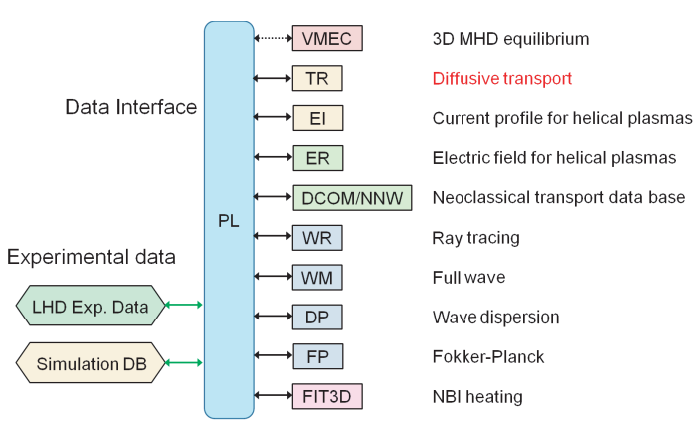

Fig. 1 Modular structure of TASK3D.

evolutions and steady-state values of the temperature and density profiles using a theoretical and/or semi-empirical transport model. The transport model, that predicts profiles consistent with experiments, is considered most suitable.

However, predictive codes such as TASK3D require a lengthy calculation time period to reproduce an experimentally measured plasma profile. In research based on experimental measurements, thermal transport coefficients have been evaluated on the basis of power balance analyses for many experiments, and their parametrical dependence has been investigated [4]. Therefore, we have customized TASK3D to evaluate transport coefficients on the basis of a power balance analysis of the experimentally measured profiles.

LHD's high-beta discharge at a low magnetic strength would be a lot of re-entering fast ions, which pass over 


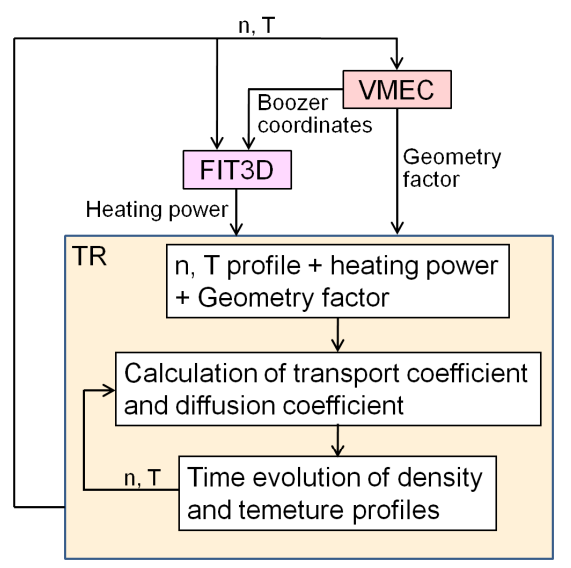

Fig. 2 TR module.

the last closed flux surface (LCFS) and repeatedly pass in and out of the LCFS. These re-entering fast ions greatly affect the heating efficiency of NBIs [5]. Therefore, we have investigated their effect on the transport coefficient as the first application of customized TASK3D.

\section{Transport Coefficient Evaluation Tool for Experimentally Measured Plasma Profiles (TR-snap)}

In this study, the one-dimensional diffusive transport module (TR module) included in TASK3D is customized in order to add a new function, TR-snap, for evaluating transport coefficients on the basis of a power balance analysis.

\subsection{TR module}

In TASK3D, the TR module is used to predict the time evolution of the temperature and density profiles due to the neo-classical transport and/or anomalous transport. The TR module numerically solves the following transport equations.

$$
\begin{aligned}
& \frac{1}{V^{\prime}} \frac{\partial\left(n_{j} V^{\prime}\right)}{\partial t}=-\frac{1}{V^{\prime}} \frac{\partial}{\partial \rho}\left(V^{\prime} \Gamma_{j}\right)+S_{j}, \\
& \frac{1}{V^{\prime} 5 / 3} \frac{\partial}{\partial t}\left(\frac{3}{2} n_{j} T_{j} V^{\prime 5 / 3}\right)=-\frac{1}{V^{\prime}} \frac{\partial}{\partial \rho}\left(V^{\prime} Q_{j}\right)+P_{j},
\end{aligned}
$$

where $n_{j}$ and $T_{j}$ are the density and temperature of species " $j$ ", respectively. $\rho$ denotes the minor radius, and $V^{\prime}=$ $\partial V / \partial \rho$. Here, $S_{j}$ and $P_{j}$ are the particle source and the heat source, respectively. The heat sources for an ion and an electron are given by

$$
\begin{aligned}
& P_{e}=-\frac{n_{e} T_{e}}{\tau_{e i}}+\frac{n_{i} T_{i}}{\tau_{i e}}+P_{\mathrm{nb}_{-} e}-P_{\mathrm{RL}}-P_{\mathrm{IE}}, \\
& P_{i}=\frac{n_{e} T_{e}}{\tau_{e i}}-\frac{n_{i} T_{i}}{\tau_{i e}}+P_{\mathrm{nb}_{-} i}-P_{\mathrm{CX}},
\end{aligned}
$$

respectively. Here, $P_{\mathrm{nb}}$ denotes the heating power of NBs, $P_{\mathrm{RL}}$ and $P_{\mathrm{IE}}$ are the radiation loss and ionization loss due to impurity ions, respectively, and $P_{\mathrm{CX}}$ is the energy loss

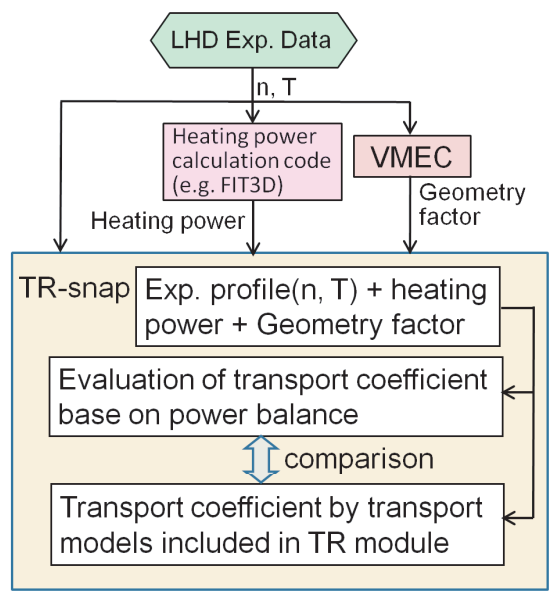

Fig. 3 TR-snap.

due to the charge exchange reaction with neutral particles. The particle flux $\Gamma_{j}$ and the heat flux $Q_{j}$ in Eqs. (1) and (2) are given by

$$
\begin{aligned}
& \Gamma_{j}=-\left\langle\left|\nabla \rho^{2}\right|\right\rangle D_{j} \frac{\partial n_{j}}{\partial \rho}+\langle\nabla \rho\rangle v_{j} n_{j}, \\
& Q_{j}=-\left\langle\left|\nabla \rho^{2}\right|\right\rangle \chi_{j} n_{j} \frac{\partial T_{j}}{\partial \rho}+\langle\nabla \rho\rangle u_{j} n_{j} T_{j}+\frac{3}{2} \Gamma_{j} T_{j},
\end{aligned}
$$

where $v_{j}$ is the particle pinch and $u_{j}$ is the thermal pinch. In the TR module, the diffusive coefficient $D$ and the thermal transport coefficient $\chi$ in Eqs. (5) and (6) are calculated by the neo-classical transport and/or anomalous transport models.

In a simulation of the plasma maintained by NBIs, the equilibrium magnetic field is calculated from the temperature and density profiles by using Variational Moments Equilibrium Code (VMEC) [6], and the heating power of the NBIs is evaluated by FIT3D [7]. Then, the time evolution of the temperature and density is calculated using these heating powers and the equilibrium magnetic field (Fig. 2).

\subsection{TR-snap}

The TR-snap function added to the TR module evaluates the transport coefficient on the basis of power balance analysis of experimentally measured profiles.

TR-snap assumes a steady-state plasma in which the heating power balances the energy loss; in such cases the time derivative term on the left side of Eq. (2) becomes zero. Thus, the thermal transport coefficients are given as

$$
\chi_{j}=-\frac{\int P_{j} V^{\prime} \mathrm{d} \rho-\langle|\nabla \rho|\rangle V^{\prime} u_{j} n_{j} T_{j}-\frac{3}{2} V^{\prime} \Gamma_{j} T_{j}}{\left\langle\left|\nabla \rho^{2}\right|\right\rangle V^{\prime} n_{j} \frac{\partial T_{j}}{\partial \rho}} .
$$

The geometry factor of the equilibrium magnetic field and the heating power are required in TR-snap. Using the experimentally measured temperature and density profiles, 


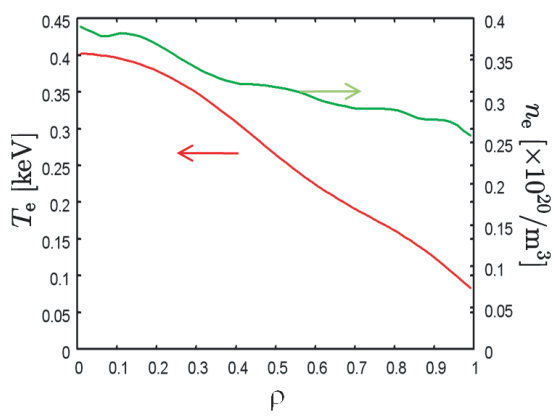

Fig. 4 Electron density and temperature.

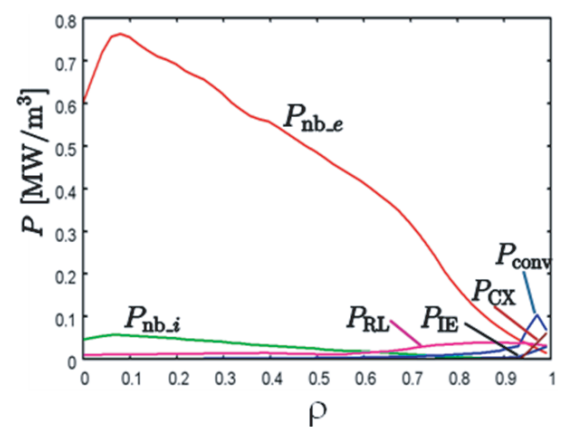

Fig. 5 Heating power and energy loss due to the impurity ions and neutrals. $P_{\mathrm{nb}_{-} e}$ and $P_{\mathrm{nb}_{-} i}$ are the heating powers of NBIs for electrons and ions, respectively. $P_{\mathrm{RL}}$ and $P_{\mathrm{IE}}$ are the radiation loss and ionization loss due to the impurity ions, respectively. $P_{\mathrm{CX}}$ denotes the charge exchange loss with neutrals, and $P_{\text {conv }}$ is the convection term. $P_{\mathrm{RL}}, P_{\mathrm{IE}}$, $P_{\mathrm{CX}}$ and $P_{\text {conv }}$ are calculated by PROCTR.

the geometry factor is calculated by VMEC, and the heating power is calculated by a heating power calculation code, e.g., FIT3D. Then, the thermal transport coefficients are evaluated from Eq. (7) using the geometry factor and the heating power (Fig. 3). In TR-snap, the thermal transport coefficients based on the power balance analysis can be compared with those obtained by transport models in TASK3D.

\section{Test Calculation Using TR-snap}

We evaluated the thermal transport coefficients in a typical LHD high-beta plasma as a test calculation using TR-snap. A high-beta plasma maintained by tangentialNBIs $\left(B_{\mathrm{ax}}=0.425 \mathrm{~T}\right.$, gamma $=1.20$ and $\left.\left\langle\beta_{\mathrm{dia}}\right\rangle=4.7 \%\right)$ was used for the test calculation; the electron density and temperature in this plasma are shown in Fig. 4. Here, gamma is the coil pitch (gamma $=m a_{\mathrm{c}} / l R_{\mathrm{c}}$, where $m$ and $l$ are the toroidal and helical pitches, respectively, and $R_{\mathrm{c}}$ and $a_{\mathrm{c}}$ are major and minor radii, respectively, of the helical coil).

In this calculation, the heating power is calculated by a simple heat power evaluation code, FIT3D. Figure 5 shows the heating power, the radiation and ionization losses due to the impurity ions, the charge exchange losses due to

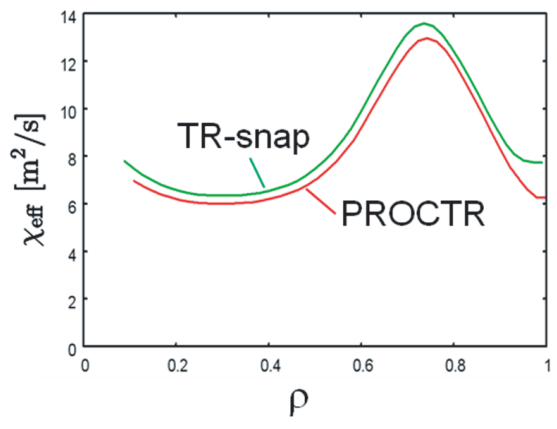

Fig. 6 Effective thermal transport coefficient.

neutrals, and the convection term. Here, the convection term denotes the particle flux due to neo-classical diffusion. The energy losses and the convection term are calculated by PROCTR. Figure 5 shows that the heating power for electrons is much larger than the convection term and the energy loss due to the impurity ions and neutrals. Thus, in this study, the thermal transport coefficient is evaluated without considering the convection term and the energy losses, and it is calculated on the basis of the measured electron temperature $\left(T_{e}\right)$ and density $\left(n_{e}\right)$, assuming that $T_{i}=T_{e}$ and $n_{i}=n_{e}$. Then, the effective thermal transport coefficient is given as [4]

$$
\chi_{\mathrm{eff}}=\frac{\chi_{e}+\chi_{i}}{2}
$$

The effective thermal transport coefficient evaluated by TR-snap is shown in Fig. 6. The result of PROCTR [8], which is used as the transport analysis code in the LHD, is also shown for reference. PROCTR is based on a power balance analysis and was developed at Oak Ridge National Laboratory. In Fig. 6, the thermal transport coefficient obtained by TR-snap is slightly larger than that obtained by PROCTR. This is because PROCTR's thermal transport coefficient including the energy loss due to the impurity ions and neutrals. Except for this difference, the value and shape of the two thermal transport coefficients are almost the same.

\section{Effect of Re-entering Fast Ions on Thermal Transport Coefficient}

As described in Sec. 1, the effect of the re-entering fast ions on the heating efficiency of the NBIs is large in LHD's high-beta plasma. Thus, the re-entering fast ions may affect the thermal transport coefficient. However, the plasma confinement property is studied on the basis of the heating power without considering re-entering fast ions. Therefore, the tendency of this property might be different if the re-entering fast ions are considered.

We investigated the effect of the re-entering fast ions on the thermal transport coefficient as the first application of customized TASK3D. To calculate the heating power with the fast ions, we used a Monte-Carlo code based on orbit following in real coordinates for helical systems 


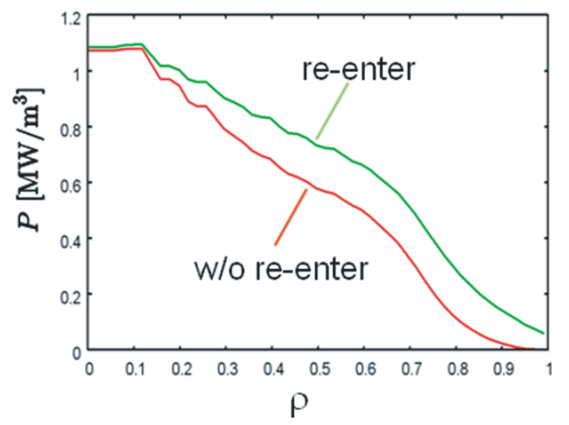

Fig. 7 Effect of re-entering fast ions on the heating power.

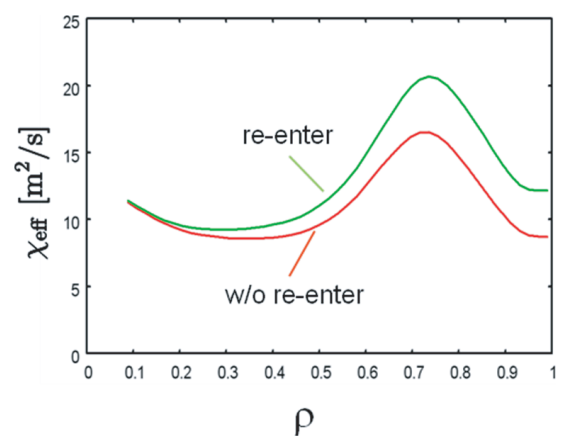

Fig. 8 Effect of re-entering fast ion on the effective thermal transport coefficient.

\section{(MORH) [5].}

Figure 7 shows the heating power calculated by MORH in the typical high-beta plasma shown in Sec. 3. The heating power with the re-entering fast ions is larger than that without them over almost the entire area.

The effective thermal transport coefficient [Eq. (8)] using the heating power evaluated by MORH is shown in Fig. 8. The difference between the coefficients is small in the center, whereas it is larger near the LCFS. The local thermal transport coefficient including the re-entering fast ions near the LCFS is 30\% larger than that without them.

Based on these results, we estimated how much the previously identified confinement property changes when the re-entering fast ions are included in the analysis. Figure 9 shows the local thermal transport coefficient near the LCFS in experiments [2]. The open circles denote the local thermal transport coefficient without re-entering fast ions, and the filled circles represent that with re-entering fast ions. In a typical LHD high beta discharge maintained by tangential-NBIs, the central electron density $\left(n_{e 0}\right)$ is $2 \times 10^{19}-4 \times 10^{19} \mathrm{~m}^{-3}$ [9]. In addition to the analysis for $n_{e 0} \simeq 4 \times 10^{19}$ shown in Fig. 8, the re-entering fast ion effect is estimated for $n_{e 0} \simeq 3 \times 10^{19}$ and $n_{e 0} \simeq 2 \times 10^{19}$ $\left(B_{\mathrm{ax}}=0.9\right)$. The thermal transport coefficient with the reentering fast ions is slightly larger than the previously identified known thermal transport coefficient. However, the difference between the two is almost the same as the dispersion of the previously identified thermal transport co-

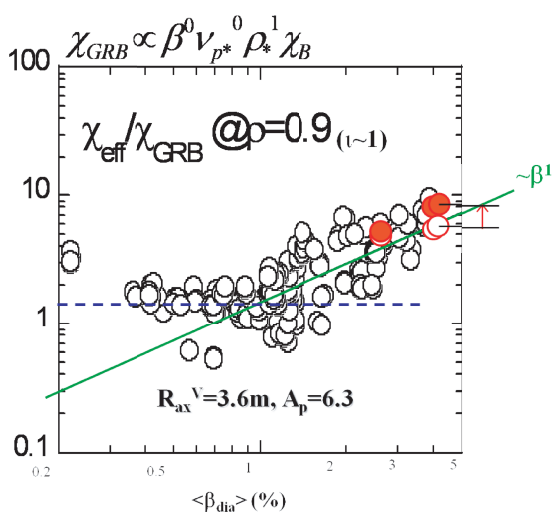

Fig. 9 Local thermal transport coefficient near LCFS in experiments [2]. The open circle denotes the hitherto known local thermal transport coefficient without re-entering fast ions, and the filled circle is the local thermal transport coefficient with re-entering fast ion. The solid and broken lines denotes $\beta^{1}$ dependency and $\beta^{0}$ dependency, respectively.

efficient. In addition, because the difference between the drift surface of the fast ions produced by tangential-NBIs and the flux surface at its birth point depends strongly on the magnetic field strength, the effect of the re-entering fast ions produced by tangential-NBIs depends on the magnetic field strength than on the beta value [5]. Low-beta experiments are conducted at a higher magnetic field compared with that used for high-beta experiments. The number of the re-entering fast ions decreases at high field strength. Thus, the effect of the re-entering fast ions in a low-beta discharge may be smaller than that in a high-beta discharge. Therefore, the tendency of the thermal transport coefficient with considering the re-entering fast ions is rarely different from the previously identified tendency.

\section{Summary}

A new function, TR-snap, for evaluating the transport coefficient on the basis of a power balance analysis was added to the integrated transport code for three dimensional configurations, TASK3D. To check the validity of TR-snap's results, the thermal transport coefficient evaluated in a typical LHD high-beta plasma was compared with the results of PROCTR, which is used as the transport analysis code in the LHD. The two thermal transport coefficients are almost the same. Using customized TASK3D, we investigated the effect of the re-entering fast ions on the thermal transport coefficient. The local thermal transport coefficient considering the re-entering fast ions is $30 \%$ larger than that without considering re-entering fast ions. However, the difference is almost the same as the dispersion of the previously evaluated thermal transport coefficient without the re-entering fast ions. The tendency of the thermal transport coefficient with considering the reentering fast ions is rarely different from the previously identified tendency. 
[1] K.Y. Watanabe et al., in Proc. of ITC-17 and ISWS-16 Toki I-13 (2007).

[2] K.Y. Watanabe, 52nd Annual Meeting of the APS Division of Plasma Physics, DI3 (2010).

[3] A. Fukuyama et al., Proc. of 20th IAEA Fusion Energy Conf. (Villamoura, Portugal, 2004) IAEA-CSP-25/CD/TH/P2-3.

[4] H. Funaba et al., Plasma Fusion Res. 3, 022 (2008).
[5] R. Seki et al., Plasma Fusion Res. 5, 027 (2010).

[6] S.P. Hirshmann et al., Comp. Phys. Comm. 43, 143 (1986).

[7] S. Murakami et al., Trans. Fusion Technol. 27, 256 (1995).

[8] H.C. Howe, ORNL/TM-11521, (1990).

[9] K.Y. Watanabe et al., Plasma Phys. Control. Fusion 49, 605 (2007). 